\title{
Peripheral Ganglioneuroblastoma
}

National Cancer Institute

\section{Source}

National Cancer Institute. Peripheral Ganglioneuroblastoma. NCI Thesaurus. Code C6594.

A gang lioneuroblastoma arising from the peripheral nervous system. 